\title{
Ultrafast electron and proton bunches correlation in laser-solid matter experiments
}

\author{
F. G. Bisesto, ${ }^{1, *}$ (1) M. Galletti, ${ }^{1}$ M. P. Anania, ${ }^{1}$ G. Costa,${ }^{1}$ M. Ferrario, ${ }^{1}$ R. Pompili,${ }^{1}$ \\ A. Poyé, ${ }^{2}$ F. Consoli, ${ }^{3}$ M. Salvadori, ${ }^{3,4,5}$ M. Cipriani, ${ }^{3}$ C. Verona, ${ }^{6}$ and A. Zigler ${ }^{1,7}$ \\ ${ }^{1}$ INFN-LNF, Via Enrico Fermi 54, 00044 Frascati, Italy \\ ${ }^{2}$ Laboratory PIIM, University Aix-Marseille-CNRS, Avenue Escadrille Normandie-Niémen, 13397 Marseille, France \\ ${ }^{3}$ ENEA Fusion and Technologies for Nuclear Safety and Security Department, C.R. Frascati, Via E. Fermi 45, 00044 Frascati, Italy \\ 'Universita' di Roma La Sapienza, Piazzale Aldo Moro 5, Roma, Italy \\ ${ }^{5}$ INRS-EMT, Varennes, Quebec J3X 1P, Canada \\ ${ }^{6}$ University of Rome Tor Vergata, Industrial Engineering Department, Via del Politecnico 1, 00133 Roma, Italy \\ ${ }^{7}$ Racah Institute of Physics, Hebrew University, 91904 Jerusalem, Israel \\ *Corresponding author: fabrizio.giuseppe.bisesto@Inf.infn.it
}

Received 16 July 2020; revised 12 August 2020; accepted 17 August 2020; posted 17 August 2020 (Doc. ID 402938); published 30 September 2020

The interaction of an ultra-intense laser with a solid state target allows the production of multi-MeV proton and ion beams. This process is explained by the target normal sheath acceleration (TNSA) model, predicting the creation of an electric field on the target rear side, due to an unbalanced positive charge. This process is related to the emission of relativistic ultrafast electrons, occurring at an earlier time. In this work, we highlight the correlations between the ultrafast electron component and the protons by their simultaneous detection by means of an electro-optical sampling and a time-of-flight diagnostics, respectively, supported by numerical simulations showing an excellent agreement. () 2020 Optical Society of America

https://doi.org/10.1364/OL.402938

The tremendous progresses in laser physics [1,2] have made it possible to overcome the technological limits hampering the study of the interaction between intense optical radiation and matter. The topic is extremely fertile and appealing for its many potential applications such as compact laser-plasma-based accelerators [3,4], sources of secondary radiation [5], mimicking astrophysical scenarios [6], and so on. The realization of chirped pulse amplification (CPA), in particular, has provided ultrashort high-energy pulses, extremely useful to investigate the interaction with solid state matter at femtosecond level. Indeed, for laser intensities larger than $10^{18} \mathrm{~W} / \mathrm{cm}^{2}$ and normalized potential vector values $a_{0} \gg 1$, the interaction, occurring in the relativistic regime, opens the way to completely new, very compact proton accelerators at sub-millimeter scale [7-9]. Indeed, extremely high electric fields on the order of TV $/ \mathrm{m}[10]$ are generated on the back surface of the target, able in theory to accelerate ions and protons at hundreds of $\mathrm{MeV}$ s in a very short distance [11]. The picture of the interaction is the following. Electrons on the target's front surface are accelerated directly by the laser [12] and propagate into the bulk, leaving the target.
Meanwhile, a positive unbalanced charge is induced on the target [13]. This, in turn, rules the establishment of a quasi-static electric potential, responsible for ion acceleration [14]. Among the totality of ejected electrons, called hot electrons, the majority is stopped in the vicinity of the back surface, within a distance of the Debye length, by the abovementioned potential, re-hitting it and producing secondary electrons. A small fraction of the negatively charged particles, hereinafter called ultrafast (UF) electrons, is able to completely escape the target, overcoming the potential due to its multi-MeV energy. The energy distribution and the charge in the escaping electron bunch are linked to the hot electron temperature, which, in turn, defines the depth of the potential well at the target's rear surface (i.e., the ion energy). Retrieving the metrics of the UF electron bunch experimentally, and correlating them with the proton beam parameters in the same shot, is invaluable to obtain quantitative details of the acceleration process. Due to the typical timescale at subpicosecond level, proper diagnostics are needed to probe such phenomena. In previous works $[10,15-18]$, we have demonstrated the feasibility of a temporally resolved diagnostic, based on an electro optic sampling (EOS) technique in the spatial encoding scheme [19], to characterize the ultrafast electron emission during laser-solid matter interactions, allowing to retrieve the ultrafast electron beam charge $\left(Q_{u f}\right)$ and temporal length $\left(\tau_{u f}\right)[16]$.

In this Letter, we experimentally demonstrate that the charge and length of the ultrafast electron bunch are in direct correlation with the proton energy gain. Qualitatively, this correlation arises from the fact that the measured parameters of the ultrafast electron bunch are defined by the temperature of hot electron distribution. The latter, in turn, defines the depth of the potential well at the target's rear surface (i.e., the proton energy.) Concerning the characterization of accelerated protons, time-of-flight (TOF) diamond detectors have been employed to retrieve their energy spectrum after the acceleration process 
[2021]. In addition to previous results, the concurrent correlation between the ultrafast electron parameters, namely, charge and temporal length, measured by EOS diagnostics and the maximum protons energy, retrieved from the implemented TOF diamond detector, is obtained as a function of the laser energy. The experimental findings are supported by means of numerical simulations by using an electron ballisticpropagation code (EBC [22]). From these, the correlations between the ultrafast electron component charge and temporal length and the established potential involved in the target normal sheath acceleration (TNSA) process are retrieved. The experiment has been performed at SPARC_LAB Test Facility employing the 100 TW laser FLAME [23].

Figure 1 shows a sketch of our setup. For this experiment, the $4 \mathrm{~J} 30$ fs laser pulses, exiting the compressor chamber, are focused onto a $10 \mu \mathrm{m}$ thin aluminium target by a $f / 10$ off-axis parabolic mirror down to $15 \mu \mathrm{m}$ waist. Thus, since $50 \%$ of energy is in the focal spot, a peak intensity in excess of $1 \times 10^{19} \mathrm{~W} / \mathrm{cm}^{2}$ is achieved on the target, corresponding to a normalized intensity $a_{0} \approx 3$. Moreover, the temporal contrast is $10^{-6}$ at ps level and below $10^{-9}$ at ns scale. Before the last multipass amplifier, a small portion of the laser beam is used as an auxialiary beamline [24], devoted mainly to probe laser interaction experiments. The time of arrival of the two beams in the target area is tuned by means of an optical autocorrelator. Furthermore, a motorized delay line, installed on the probe line optical path, allows to perform a temporal scan of the interaction (500 ps of travel). The probe laser is employed to detect the electric field of the ultrafast electrons escaping the target after the interaction, leaving a signature in an electro optical $\mathrm{ZnTe}$ crystal, placed $2 \mathrm{~mm}$ far from the focus and above it. As shown in our previous works $[15-17,20]$, this technique allows to reconstruct the electron longitudinal distribution from the probe laser transverse profile by means of a polarization decoding line. In our case, two polarizers (P1 and P2) are installed before and after the $\mathrm{ZnTe}$ crystal, with an extinction coefficient of $10^{-4}$. Due to the setup geometry and probe laser size, a temporal window of 8 ps with $100 \mathrm{fs}$ resolution is achieved. Due to the its position, plasma debris released during the interaction can damage the crystal. For this reason, this was constantly monitored, by means of a dedicated camera, and replaced if needed.

On the other side, the proton bunches, accelerated due to the extremely high $(\sim \mathrm{TV} / \mathrm{m})$ electric field present on the target's

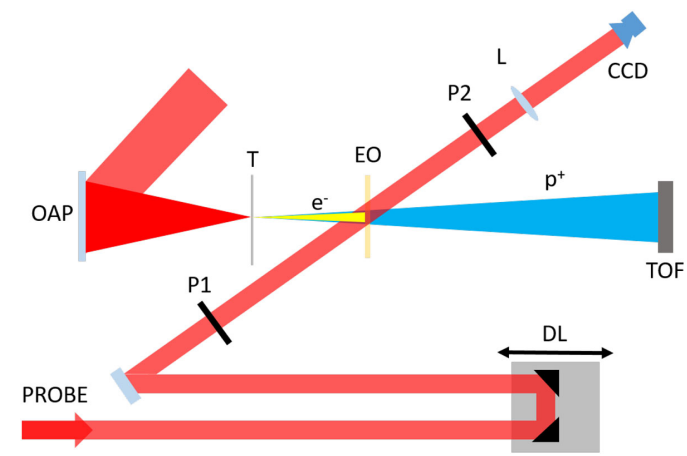

Fig. 1. FLAME laser is focused by a parabola (OAP) and sent onto a $10 \mu \mathrm{m}$ thick aluminum target (T). An electro-optical sampling diagnostic, relying on two polarizers (P1 and P2), a ZnTe crystel (EO), and an imaging lens (L), exploiting a delay line (DL) to temporally scan the interaction, and a time-of-flight diamond detector (TOF) have been installed. rear surface [10], was measured by means of a TOF diamond detector, placed $1 \mathrm{~m}$ far from the target, at $0^{\circ}$ with respect to the laser's incident direction and having a line of sight of the target completely free. The diamond detector was fabricated in a planar interdigitized electrode configuration, allowing to reach a temporal resolution less than 800 ps [21,25], resulting in an energy resolution below $1 \%$ in the $\mathrm{MeV}$ range. On the other hand, the signal coming from the TOF detector cannot be related to any possible carbon ion. In the presented experiments, the diamond detector was covered with a $10 \mu \mathrm{m}$ aluminum foil, able to stop carbon ions with energies $\leq 12 \mathrm{MeV}$. According to our experimental parameters, this is very much beyond what is expected for $\mathrm{C}$ ions. Our experiment is in common TNSA conditions, where ions are accelerated by a potential sheath developed on the rear surface of the target. They will thus gain kinetic energy according to this potential and their average charge. Since protons have the highest charge-to-mass ratio with respect to other ions, for a given potential, they will be the first to reach the detector. In a TOF detection scheme, the first signal observed will be thus clearly due to the most energetic protons, very much separated in time with respect to other possible energetic ion components. Moreover, in this Letter, we report results of just the maximum proton energy, rather than the whole proton spectra, and thus no contribution coming from $\mathrm{C}$ or other ion species can be present. Figure 2 shows the ultrafast electron charge value measured for different laser energies. The error bars represent the statistical error. Fitting the experimental data with a power law, the relation between electrons' charge and laser energy has been found to be $Q_{u f}[n C]=(0.59 \pm 0.03) \times\left(E_{L}[J]\right)^{(0.75 \pm 0.01)}$. The result is supported by models $[8,26,27]$ in literature, describing intense laser-solid matter interaction. In detail, assuming the number of ultrafast electrons is defined as $N_{u f}=\delta * N_{\text {hot }}$, with $\delta \ll 1$ [10], i.e., the ultrafast electrons are a small portion of the hot electrons population, $N_{\text {hot }}=\eta * E_{L} / E_{\text {hot }}$, where $\eta \propto E_{L}^{1 / 4}$ is the photons-to-hot electrons conversion efficiency coefficient, $E_{L}$ is the laser energy, and $E_{\text {hot }}=m_{e} c^{2} \times\left\{\left(1+a_{0}^{2}\right)^{0.5}-1\right\}$, following the ponderomotive scaling law [22], is the average hot electron energy [13]; since $a_{0} \propto E_{L}^{0.5}$, one finds $Q_{u f} \propto E_{L}^{0.75}$. For each EOS signal, also the proton energy was measured by means of a TOF diamond detector. Figure 2 reports how the highest measured proton energy behaves as a function of laser energy. As expected, increasing the number of photons hitting the target, protons leave it with higher energy. Indeed, we found experimentally

$$
E_{p}[\mathrm{MeV}]=(1.84 \pm 0.09) \times\left(E_{L}[\mathrm{~J}]\right)^{(0.56 \pm 0.12)},
$$

in agreement with previous works [28-30]. In Fig. 2, a correlation between ultrafast electrons and protons can be derived. The proton energy goes up as ultrafast electrons' extracted charge from the target increases with an almost linear dependence. This is a direct consequence of a stronger potential established on the target's rear surface, able to accelerate protons from ionized layers to higher energies. The experimental evidence of correlation between proton energy and ultrafast electron charge confirms the guess we have done in a previous work [15], where different target geometries were studied. In particular, it was claimed that for shorter and higher charged ultrafast electron bunches, the expected proton energy is supposed to increase. Furthermore, the electrons leaving the target are characterized by a higher energy, and thus they present a shorter bunched structure. 


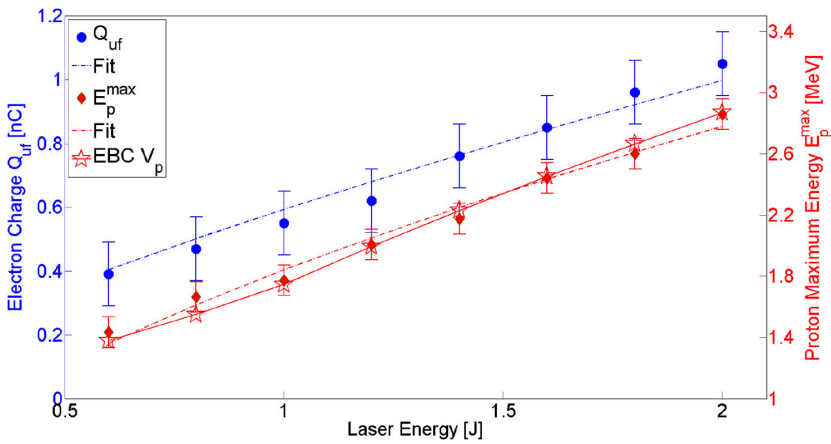

Fig. 2. Experimental ultrafast electrons charge versus proton maximum energy for different laser energies.

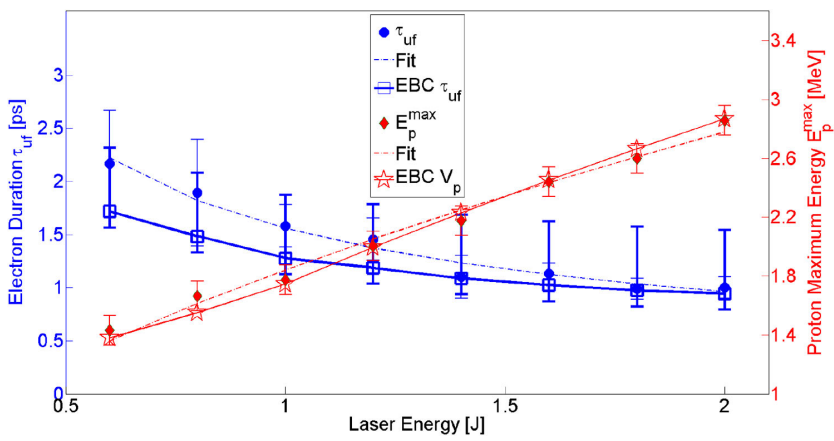

Fig. 3. Experimental and simulated ultrafast electrons temporal duration versus proton maximum energy for different laser energies.

In Fig. 3, a different correlation between UF electrons and protons can be derived. The larger error bars at lower laser energy are due to a lower SNR, since less charge is extracted and a weaker field is detected. The measured proton energy goes up with the increase in laser energy. This is correlated to the shortening of the measured electron bunch as highligthed by the retrieved power law $\tau_{u f}[\mathrm{ps}]=(1.56 \pm 0.07) \times\left(E_{L}[\mathrm{~J}]\right)^{(-0.70 \pm 0.1)}$. The latter can explained by a smaller velocity spread when $\beta \sim 1$ at higher laser energies. As a consequence, shorter highly charged electron beams suggest a stronger accelerating potential at the target's rear surface. The experimental measurements related to the UF electron components can be linked directly to the retrieved proton energy, as shown in Fig. 4. Here, we show the maximum proton energy as a function of the UF electron component charge. Fitting the experimental data with a power law, an empirical law has been retrieved:

$$
E_{p}[\mathrm{MeV}]=2.7 \pm 0.2 \times\left(Q_{u f}[\mathrm{nC}]\right)^{(0.6 \pm 0.1)} .
$$

Moreover, due to the adopted temporally resolved diagnostic, we were also able to extrapolate the relation between the maximum proton energy and the UF electron component duration. Fitting the experimental data with a power law, an empirical law has been retrieved: $E_{p}[\mathrm{MeV}]=2.8 \pm 0.1 \times$ $\left(\tau_{u f}[\mathrm{ps}]\right)^{(-0.9 \pm 0.2)}$. We have to highlight that this relation is valid in our laser energy range leading to ps-long bunches. Further studies should be performed at higher laser energy aiming at shorter bunches at fs level. Finally, these empirical laws show the foreseen correlation between the electron and

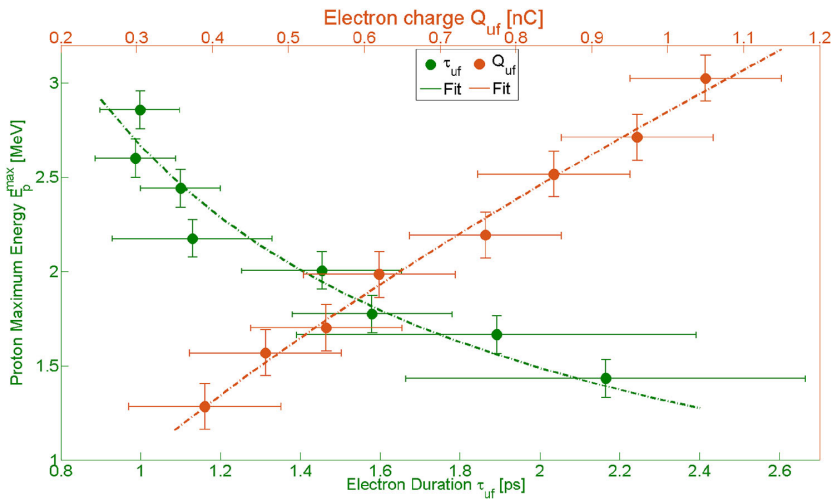

Fig. 4. Proton maximum energy as a function of the ultrafast electron duration and charge.

proton beams ruled by the deduced quasi electro static potential. As pointed out above, the detection of shorter highly charged electron beams suggests the creation of a stronger potential on the target's rear side, while increasing the laser energy, allowing the production of more energetic protons. The retrieved experimental data regarding characterization of the UF energetic electron component, linked strictly to the potential barrier $V_{p}$ [31], in turn responsible for the proton acceleration, are supported by the EBC [22]. Due to this model, we can first retrieve the early potential by applying

$$
V_{p}=T_{h}\left(\frac{\lambda}{\sqrt{\lambda^{2}+1}} \log \left(\frac{\sqrt{\lambda^{2}+1}+1}{\lambda *\left(\sqrt{\lambda^{2}+1}-\lambda\right)}\right)-\log (2 \lambda)\right) .
$$

From here, the charge of electrons reaching the rear side with energies $\varepsilon \geq V_{p}$ reads

$$
Q_{u f} \simeq \int_{V_{p}}^{\infty} \eta\left(I_{L}\right) \frac{e \Delta E_{L} \exp \left(-\varepsilon / T_{h}\right)}{t_{L}} \times\left(\frac{1}{v_{V_{p}}}-\frac{1}{v(\varepsilon)}\right) \mathrm{d} \varepsilon,
$$

where $\lambda=\sqrt{2 * \exp (1)} \lambda_{D} / r_{L}$, with the Debye length defined as $\lambda_{D}=\sqrt{\varepsilon_{0} T_{b} / e^{2} n}$, where $n=N_{h} e / \pi r_{L}^{2} t_{L} \int_{0}^{\infty} v(\varepsilon)$ $\exp \left(-\varepsilon / T_{h}\right) \mathrm{d} \varepsilon$ is the electron density, $r_{L}$ and $t_{L}$ are the laser radius and duration, respectively, $\varepsilon$ is the electron energy, $v(\varepsilon)$ is the electron speed, and $\Delta$ is the target thickness. For our purposes, we used $N_{\varepsilon}=5 e^{3}$ points to discretize the energy, assumed $E_{\max }=20 T_{b}$ to be its maximum value, and $0.01 \mathrm{fs}$ as the time unit. The simulation data regarding the charge $Q_{u f}[$ Eq. (4)] and the magnitude of the potential barrier $V_{p}$ [Eq. (3)], as a function of the laser energy, are reported in Fig. 2 showing an excellent agreement with the experimental results. It is worth to stress that these results refer only to the UF component of the emitted particles and not to the total population, as done in other works [32-34], whose charge according to our simulations ranges from $\sim 37 \mathrm{nC}$ to $\sim 110 \mathrm{nC}$. Furthermore, we used the EBC to explain also the measured UF electron beam duration [22].The results for $\tau_{u f}$ are shown in Fig. 3. In detail, for larger laser intensities, the ejected relativistic electrons present a small velocity spread, resulting in a shorter bunch; meanwhile, as the laser energy decreases, the electron velocity spread increases, resulting in longer bunches. Nevertheless, the difference between our 
model and data increases in the case of lower laser energies. The reason is twofold. On one hand, the EOS signal is affected by the electron energy: at lower energy, the same signal looks wider because of the field opening in the longitudinal direction $\left(\propto r / \gamma_{e}\right)$ [16]. On the other hand, some assumptions in our model, such as the energy cut at $V_{p}$ and the absence of some collective effects, e.g., space charge forces $\left(\propto \gamma_{e}^{-2}\right)$, are negligible only for relativistic particles, leading to an additional contribution in temporal broadening. In conclusion, we have shown in our Letter how the emission of ultrafast electrons and protons, produced in the same ultra-intense laser-solid matter interaction, changes by varying the laser energy on the target. In detail, two different detectors, an EOS and a TOF diamond, have been employed to measure the charge and duration of the ultrafast electrons and the proton energy spectrum, respectively. From our data, we found that as the laser energy increases, more charged and shorter ultrafast electron bunches are detected. In turn, a higher electric field is responsible for more energetic protons released from the target, as highlighted by the proton energy measurement. Moreover, we have shown for the first time two empirical laws [Eqs. (1) and (2)] linking the measured ultrafast electron properties with the proton maximum energy for our experimental parameters. Our measurements have been supported by numerical simulations from the EBC, a simplified scalar model complementary to the well-known ChoCoLaTII, showing an excellent agreement. In detail, it allowed us to determine the potential, established on the target real surface, responsible for proton acceleration, relating it to electron beam charge and duration.

Disclosures. The authors declare no conflicts of interest.

\section{REFERENCES}

1. C. Danson, D. Hillier, N. Hopps, and D. Neely, High Power Laser Sci. Eng. 3, e3 (2015).

2. C. N. Danson, C. Haefner, J. Bromage, T. Butcher, J.-C. F. Chanteloup, E. A. Chowdhury, A. Galvanauskas, L. A. Gizzi, J. Hein, D. I. Hillier, N. Hopps, Y. Kato, E. Khazanov, R. Kodama, G. Korn, R. Li, Y. Li, J. Limpert, J. Ma, C. H. Nam, D. Neely, D. Papadopoulos, R. Penman, L. Qian, J. Rocca, A. Shaykin, C. W. Siders, C. Spindloe, S. Szatmári, R. Trines, J. Zhu, P. Zhu, and J. D. Zuegel, High Power Laser Sci. Eng. 7, e54 (2019).

3. S. Steinke, J. Van Tilborg, C. Benedetti, C. Geddes, C. Schroeder, J. Daniels, K. Swanson, A. Gonsalves, K. Nakamura, N. Matlis, B. H. Shaw, E. Esarey, and W. P. Leemans, Nature 530, 190 (2016).

4. E. L. Clark, K. Krushelnick, J. R. Davies, M. Zepf, M. Tatarakis, F. N. Beg, A. Machacek, P. A. Norreys, M. I. K. Santala, I. Watts, and A. E. Dangor, Phys. Rev. Lett. 84, 670 (2000).

5. A. Rousse, K. T. Phuoc, R. Shah, A. Pukhov, E. Lefebvre, V. Malka, S. Kiselev, F. Burgy, J.-P. Rousseau, D. Umstadter, and D. Hulin, Phys. Rev. Lett. 93, 135005 (2004).

6. A. Rigby, F. Cruz, B. Albertazzi, R. Bamford, A. R. Bell, J. E. Cross, F. Fraschetti, P. Graham, Y. Hara, P. M. Kozlowski, Y. Kuramitsu, D. Q. Lamb, S. Lebedev, J. R. Marques, F. Miniati, T. Morita, M. Oliver, B. Reville, Y. Sakawa, S. Sarkar, C. Spindloe, R. Trines, P. Tzeferacos, L. O. Silva, R. Bingham, M. Koenig, and G. Gregori, Nat. Phys. 14, 475 (2018).

7. S. Wilks, A. Langdon, T. Cowan, M. Roth, M. Singh, S. Hatchett, M. Key, D. Pennington, A. MacKinnon, and R. Snavely, Phys. Plasmas 8, 542 (2001).

8. M. Roth, T. Cowan, M. Key, S. Hatchett, C. Brown, W. Fountain, J. Johnson, D. Pennington, R. Snavely, S. Wilks, K. Yasuike, H. Ruhl, F. Pegoraro, S. V. Bulanov, E. M. Campbell, M. D. Perry, and H. Powell, Phys. Rev. Lett. 86, 436 (2001).
9. H. Schwoerer, S. Pfotenhauer, O. Jäckel, K.-U. Amthor, B. Liesfeld, W. Ziegler, R. Sauerbrey, K. Ledingham, and T. Esirkepov, Nature 439, 445 (2006).

10. R. Pompili, M. Anania, F. Bisesto, M. Botton, E. Chiadroni, A. Cianchi, A. Curcio, M. Ferrario, M. Galletti, Z. Henis, M. Petrarca, E. Schleifer, and A. Zigler, Sci. Rep. 8, 3243 (2018).

11. B. A. Remington, D. Arnett, R. Paul, and H. Takabe, Science 284, 1488 (1999).

12. P. K. Singh, Y. Cui, G. Chatterjee, A. Adak, W. Wang, S. Ahmed, A. D. Lad, Z. Sheng, and G. R. Kumar, Phys. Plasmas 20, 110701 (2013).

13. A. Poyé, J.-L. Dubois, F. Lubrano-Lavaderci, E. D’Humières, M. Bardon, S. Hulin, M. Bailly-Grandvaux, J. Ribolzi, D. Raffestin, J. Santos, Ph. Nicolaï, and V. Tikhonchuk, Phys. Rev. E 92, 043107 (2015).

14. A. Macchi, M. Borghesi, and M. Passoni, Rev. Mod. Phys. 85, 751 (2013).

15. R. Pompili, M. Anania, F. Bisesto, M. Botton, M. Castellano, E. Chiadroni, A. Cianchi, A. Curcio, M. Ferrario, M. Galletti, Z. Henis, M. Petrarca, E. Schleifer, and A. Zigler, Sci. Rep. 6, 35000 (2016).

16. R. Pompili, M. Anania, F. Bisesto, M. Botton, M. Castellano, E. Chiadroni, A. Cianchi, A. Curcio, M. Ferrario, M. Galletti, Z. Henis, M. Petrarca, E. Schleifer, and A. Zigler, Opt. Express 24, 29512 (2016).

17. F. Bisesto, M. P. Anania, M. Botton, E. Chiadroni, A. Cianchi, A. Curcio, M. Ferrario, M. Galletti, R. Pompili, and E. Schleifer, Quantum Beam Sci. 1, 13 (2017).

18. M. Galletti, F. Bisesto, M. P. Anania, M. Ferrario, R. Pompili, A. Poyé, and A. Zigler, Opt. Lett. 45, 4420 (2020).

19. A. L. Cavalieri, Electro-Optic Characterization of Femtosecond Electron Bunches (University of Michigan, 2005).

20. F. Bisesto, M. Galletti, M. P. Anania, M. Ferrario, R. Pompili, M. Botton, E. Schleifer, and A. Zigler, High Power Laser Sci. Eng. 7, e56 (2019).

21. F. Bisesto, M. Galletti, M. Anania, M. Ferrario, R. Pompili, M. Botton, A. Zigler, F. Consoli, M. Salvadori, P. Andreoli, and C. Verona, High Power Laser Sci. Eng. 7, e53 (2019).

22. M. Galletti, F. G. Bisesto, M. P. Anania, M. Ferrario, R. Pompili, A. Poyé, V. Tikhonchuk, and A. Zigler, Appl. Phys. Lett. 116, 064102 (2020).

23. F. Bisesto, M. Anania, M. Bellaveglia, E. Chiadroni, A. Cianchi, G. Costa, A. Curcio, D. Di Giovenale, G. Di Pirro, M. Ferrario, F. Filippi, A. Gallo, A. Marocchino, R. Pompili, A. Zigler, and C. Vaccarezza, "The flame laser at SPARC_LAB," arXiv:1802.00398v1 (2018) Nucl. Instrum. Methods Phys. Res. A.

24. M. Galletti, C. Coyle, P. Oliveira, M. Galimberti, F. Bisesto, and D. Giulietti, J. Instrum. 14, C02005 (2019).

25. M. Marinelli, E. Milani, G. Prestopino, and C. Verona, Appl. Surf. Sci. 272, 104 (2013).

26. S. Wilks, W. Kruer, M. Tabak, and A. Langdon, Phys. Rev. Lett. 69, 1383 (1992).

27. J.-L. Dubois, F. Lubrano-Lavaderci, D. Raffestin, J. Ribolzi, J. Gazave, A. C. La Fontaine, E. d'Humières, S. Hulin, P. Nicolaï, A. Poyé, and V. T. Tikhonchuk, Phys. Rev. E 89, 013102 (2014).

28. K. Zeil, S. Kraft, S. Bock, M. Bussmann, T. Cowan, T. Kluge, J. Metzkes, T. Richter, R. Sauerbrey, and U. Schramm, New J. Phys. 12, 045015 (2010).

29. J. Fuchs, P. Antici, E. d'Humières, E. Lefebvre, M. Borghesi, E. Brambrink, C. Cecchetti, M. Kaluza, V. Malka, M. Manclossi, S. Meyroneinc, P. Mora, J. Schreiber, T. Toncian, H. Pépin, and P. Audebert, Nat. Phys. 2, 48 (2006).

30. Y. Oishi, T. Nayuki, T. Fujii, Y. Takizawa, X. Wang, T. Yamazaki, K. Nemoto, T. Kayoiji, T. Sekiya, and A. A. Andreev, Phys. Plasmas $\mathbf{1 2}$ 073102 (2005).

31. J. J. Santos, A. Debayle, P. Nicolaï, V. Tikhonchuk, M. Manclossi, D. Batani, A. Guemnie-Tafo, J. Faure, V. Malka, and J. J. Honrubia, Phys. Plasmas 14, 103107 (2007).

32. K. Kanaya and S. Okayama, J. Phys. D 5, 43 (1972).

33. E. Aktan, H. Ahmed, B. Aurand, M. Cerchez, A. Poyé, P. Hadjisolomou, M. Borghesi, S. Kar, O. Willi, and R. Prasad, Phys. Plasmas 26, 070701 (2019).

34. M. Thévenet, A. Leblanc, S. Kahaly, H. Vincenti, A. Vernier, F. Quéré, and J. Faure, Nat. Phys. 12, 355 (2016). 